\title{
LOS CELTAS Y LA HISTORIA
}

\author{
Por \\ BEATRIZ DÍAZ SANTANA ${ }^{1}$
}

\section{RESUMEN}

El objetivo de este trabajo es analizar el concepto de Historia en la investigación protohistórica gallega, poniéndolo en relación con la evolución del concepto celta. El marco cronológico de estudio abarca desde el s. XIX hasta el presente. La Historia se concibe aquí en su sentido de elaboración historiográfica sobre el hecho histórico. Desde el s. XIX hasta el presente, se ha producido un paulatino decrecimiento del nivel de aceptación de la subjetividad y la utilidad social inherentes al concepto de Historia, que han sido retomados por el postprocesualismo en los últimos años. La Historia ha pasado de ser considerada una herramienta social y políticamente productiva en el s. XIX a enfatizar su especialización científica y aislamiento respecto a la vida social que la rodea. Esto se corresponde con cambios en sus esferas de difusión, tanto más reducidas cuanto mayor ha sido su nivel de profesionalización.

\section{PALABRAS CLAVE}

Galicia; Historia; Historiografía; protohistoria; celta; subjetivismo; difusión.

${ }^{1}$ Dpto. de Prehistoria. Facultad de Geografía e Historia, U.C.M. 28040 Madrid. Email: Beatriz_ds@hotmail.com.

«CUADERNOS DE ESTUDIOS GALLEGOS», Tomo XLVIII, Fascículo 114, Santiago 2001. 


\begin{abstract}
My aim is no other than studying the evolution of the concept of History in proto-historic Galician research, from the XIX th. century onwards. The word History is taken as the historiographic research process and production. Subjectivism and social profit of History have been avoided, as long as History has improved its specialization and scientific character. The more specialized History and Archaeology are, the more reduced and corporate historical knowledge diffusion has became. The concept of celt has been chosen as a paradigmatic case study.
\end{abstract}

\title{
KEYWORDS
}

Galicia (NW. Iberian Peninsula); History; historiography; Protohistory; celt; subjectivism; diffusion.

\section{INTRODUCCIÓN}

Las ideologías dominantes en cada época, en el contexto de cada historiador, modelan las interpretaciones que se hacen a partir del registro arqueológico. Desde una postura relativista, es posible afirmar que tanto algunos temas de estudio como la orientación final que se da a las interpretaciones basculan hacia la afirmación de las asunciones ideológicas. En otras palabras, las interpretaciones históricas desde la Arqueología son coherentes, siempre, con la ideología del autor. Pero, ¿se adaptan las interpretaciones al contexto ideológico, o es éste el que va modelando y orientando, consciente o inconscientemente, los temas de estudio y sus interpretaciones?

El punto de inflexión en este trabajo será la valoración del grado de consciencia de esta orientación ideológica en diferentes investigadores sobre la Protohistoria del territorio que actualmente ocupa Galicia. De una manera diacrónica, se pretende analizar cómo ha ido evolucionando el concepto que los diferentes historiadores de la Cultura Castreña del Noroeste de la Península Ibérica tenían de la elaboración historiográfica,

«CUADERNOS DE ESTUDIOS GALLEGOS», Tomo XLVIII, Fascículo 114, Santiago 2001. 
del proceso de investigación y análisis histórico, sobre todo respecto al celtismo y la identidad gallega. La Historia será entendida aquí como elaboración historiográfica, y no como la realidad ontológica de lo histórico (Aróstegui 1995). Mantendré esta denominación, aunque incorrecta (Ibíd.), ya que supone una aproximación constante más fácil a lo largo de todo el texto, y puesto que no trataré las bases históricas reales sobre las que se sustentan las interpretaciones de los autores aquí analizados.

\section{LA BÚSQUEDA DE UN DESTINO: LOS ROMÁNTICOS}

En el siglo XIX existía una conciencia de la utilidad social e ideológica de las ciencias, sobre todo de las ciencias humanas. La historia no se concebía como un saber independiente, desconectado de la vida social. Por el contrario, se entendía como un instrumento de cohesión social, como algo útil, tal como están haciendo los postprocesuales o los marxistas (Bate 1998) en estos momentos.

Es común encontrar en las introducciones de las numerosas Historias de Galicia que se escriben, ya desde principios del s. XIX, una declaración de las intenciones del autor cuando escribe su historia. No se concibe la investigación histórica o prehistórica si no conlleva un objetivo social o ideológico. En primer lugar, el tema de estudio, las culturas de Galicia a lo largo de toda su historia, establecieron un marco geográfico unitario. Los límites de la región del s. XIX se trasladaron al pasado. Este marco geográfico se establecía por el autor consciente y arbitrariamente en el presente, de modo que su proyección en el pasado era subjetiva y artificial. El establecimiento de este trazado casi lineal, a partir del cual la frontera de la identidad del presente se retrotraía al pasado, es explicable a partir del concepto de historia del s. XIX. Así, Verea y Aguiar, en su Historia de Galicia (1838), dice que su obra es hija del «patriotismo y del amor a la verdad histórica». La Historia tenía que ser la parte más instructiva, la más útil y agradable de la literatura, y al mismo tiempo «luz de la verdad y maestra del vivir» (op. cit., pg. 2). Junto al afán de objetividad, el autor afirma con esto que la historia tiene la finalidad práctica de dotar de sentido a la patria, encontrando dos razones de peso para escribir una Historia específica de Galicia: una la del maltrato que los historiadores españoles habían infligido a esta región, y la otra sus excelencias 
naturales, tan poco valoradas. Según este autor, la elaboración de la Historia General de una Nación es la "historia del hombre y de la naturaleza de ese país", una geografía descriptiva esquematizada en un armazón cronológico (op. cit., pg. 65 y ss.).

Benito Vicetto dice, en el prólogo de su Historia de Galicia (1865), que quiere «dotar a mi país de una historia, propiamente dicha, ya que no tiene ninguna». La elaboración de las primeras Historias de Galicia fue una respuesta a la estrategia de dominio que desde una perspectiva centralista se ejercía mediante la negación del pasado (Mansilla 1997), en este caso del pasado de las diferentes regiones con nacionalismos periféricos. La suya es una historia del pueblo, al que va dirigida y al que debe hacer ver su propia especificidad, entendida como una narración correlativa y cronológica de los sucesos prósperos o adversos que constituyeron su vida. Y, continúa, "la historia de Galicia, tal y como la concibo, por las tradiciones y los textos de los autores que me han precedido, no es la historia mísera y estéril de una localidad, de una provincia, de una nación sin influencia en el desenvolvimiento político, moral y religioso de la humanidad. La historia de Galicia es, por el contrario, la historia del primer pueblo de Europa, pues desde sus últimos ventisqueros surgió la raza más dominante y en sus últimos ventisqueros se consolidó la doctrina social más humanamente divina» (Vicetto 1865, pg.VII). Vicetto, con su estilo y su concepción narrativista de la Historia (curiosamente muy en boga entre los postprocesuales en la actualidad), quiere hacer un cuerpo de Historia para el pueblo gallego de su presente. Son los límites geográficos y culturales de ese pueblo gallego los que Vicetto traslada hacia el pasado, sin tener en cuenta las variaciones diacrónicas de la extensión y carácter de la cultura. La Historia de Vicetto, es una historia narrativa (Rojo Salgado 1997) frente a las analíticas de su época. Se diferencia de Verea, o de Martínez Padín, en que él no se considera una «superioridad intelectual», en posesión de una verdad objetiva. Mientras sus contemporáneos disfrazan su instrumentalización de la historia a través del positivismo, Vicetto admite la subjetividad de sus afirmaciones, siendo un precursor del relativismo postprocesual (Hernando 1992; Trigger 1992). Vicetto hace una Historia moral y filosófica de una manera intencionada, mientras que sus contemporáneos se suben al caballo del positivismo historicista y de la aceptación de una verdad objetiva. Lo que todos ellos tenían en común, era la asunción de la orientación práctica y

«CUADERNOS DE ESTUDIOS GALLEGOS», Tomo XLVIII, Fascículo 114, Santiago 2001. 
„sobre todo moral de la Historia. Todos ellos llegan al mismo fin por el mismo medio: la elaboración de una Historia nacional y nacionalista, la demostración de los orígenes históricos y culturales de su identidad y sus límites geográficos desde el pasado. Es el primer paso hacia la búsqueda de la identidad nacional: la búsqueda de una identidad cultural eterna e inmutable, histórica y, por lo tanto, científica.

Amparado también en el historicismo, Martínez Murguía (1888) expresa en el Discurso preliminar y sus Consideraciones, la búsqueda del Galleguismo en toda su obra. Dentro de este contexto ideológico, el autor inserta su propia elaboración histórica. Son numerosos sus análisis del concepto de nación, y la importancia que tienen las tradiciones, como la forma más visible de pervivencia histórica de la cultura y la identidad, lo mismo que Vicetto (op. cit., pg. 228). Pero Murguía no se detiene tanto como Vicetto o Verea en analizar el concepto de Historia, porque considera evidente la existencia de una verdad objetiva, como expresa en frases a lo largo de su obra: «la verdad es superior a los sistemas (de la vida contemporánea)» (op. cit., pg. 61), «la verdadera historia...» (pg.108). Un aspecto destacable es que Murguía diferencia entre la identidad del pueblo, su demostración histórica y posteriormente la creación de la identidad nacional. Tal como actualmente mantiene Gellner (1983, en Eriksen 1993: 99) Murguía distinguía entre la identidad cultural y nacional, y el nacionalismo entendiendo a éste como aquella teoría que sostiene que el fin último de la identidad y de la nación es la cristalización de éstas en una estructura política.

Y finalmente, tampoco Leandro Saralegui y Medina (1894) expone el concepto de Historia que subyace en su obra, ya que se sobreentiende que es una verdad objetiva, erudita. Para todos estos historiadores del s. XIX, Galicia es, como dice Don José Villa-amil y Castro, «el antiguo reino en que habitualmente resido» (1873: 1-66). El Celtismo de todos estos autores decimonónicos formaba parte de un discurso histórico de carácter práctico conscientemente asumido y explicitado por todos ellos tanto en sus introducciones como a lo largo de todas sus obras.

$\mathrm{Al}$ igual que en el resto de Europa, empezaban a surgir orientaciones biológicas para la explicación de las diferencias culturales. La aproximación científica al estudio de las distintas «razas» de seres humanos era una explicación y una realidad metodológica en términos históricos y biológicos (Eriksen 1993: 61). Es la época del evolucionismo, del 
historicismo y el positivismo más exacerbado (salvo en Vicetto), pero también del nacimiento de la Prehistoria como disciplina histórica, y de la Arqueología (Pereira González 1996 y 1997). Los investigadores y eruditos gallegos están dentro de las corrientes de investigación europeas, y empiezan a aparecer en sus obras las citas de los autores que han leído y en que se fundamentan, al mismo tiempo que el conocimiento de los datos de la cultura material pasa de una concepción mítica a una descripción arqueológica. Así, por ejemplo, Saralegui y Medina, opta por no creer en el evolucionismo de Cuvier para explicar al hombre «primitivo», y sí en los descubrimientos de Boucher de Perthes y de Lartet. Mientras, mantiene discursos míticos en cuanto al megalitismo, al que sigue considerando como restos de los arios celtas que habitaban la Galicia Protohistórica (1894, pg. I-VII). Estamos en los albores de la Arqueología Prehistórica, donde se confunden y entremezclan explicaciones que van quedando obsoletas y otras que las van sustituyendo.

La Arqueología como disciplina se encontraba por estas fechas en fase de profesionalización, tanto en Galicia como en el resto de España (Pasamar y Peiró, 1989/90). De manera que, dentro de los discursos históricos que se elaboraban, ahora más directamente controlados por el Estado, se planteaba el conflicto de cuáles eran los antepasados de cada nación y de cada región. Es el momento de enfatizar la relación directa entre el presente y una cultura determinada del pasado como origen de la identidad nacional y cultural. El proceso de selección es, en definitiva, arbitrario y coyuntural, y determinado en última instancia por la oposición y la validación cientifista de una frente al resto de las culturas nacionales contemporáneas y sus originarias del pasado, que pasan a ser inferiores (Collis, 1996; Díaz-Andreu 1997a). Según M. Díaz Andreu (1997: 407) el Rexionalismo gallego sólo tardíamente llega a dar lugar en el s. XX a un movimiento semejante a los observados en Cataluña y el País Vasco. Esto tendría lugar, según ella, con la creación de la Xeración Nós. Sin embargo, el proceso de selección de una época y una determinada cultura por parte de una elite intelectual, en general una elite burguesa, tuvo lugar en Galicia ya a finales del s. XIX, o apurando un poco, ya con Verea y Aguiar (1838). Desde la Historia de Galicia de Verea y Aguiar de 1838, hasta la Edade do ferro na Galiza de Florentino López Cuevillas en 1925, el carácter diferencial gallego (es decir, la identidad étnica o como pueblo de los gallegos -ver Eriksen 1993 para los procesos de creación 
de identidades étnicas en el presente) se identifica a partir de modelos teóricos historicistas, en un primer momento particularistas y difusionistas, derivando posteriormente en un positivismo de carácter más histórico cultural. Este Historicismo mantenía en los historiadores del s. XIX como objetivo teleológico la creación de una nación gallega, a través de la demostración de la existencia de la identidad gallega y de sus raíces históricas Por el contrario, durante la primera mitad del s. XX y parte de la segunda, el particularismo histórico cultural enfatizaba, al igual que lo sigue haciendo en el presente, el carácter histórico, real y objetivo de los hechos diferenciales de la identidad a la que responde, abandonando la orientación teleológica nacionalista pero justificando igualmente el presente a través del pasado. Tal como sucedía en el resto de Europa (Collis 1993; Renfrew 1996) y en el discurso histórico nacionalista más español (Jiménez Díez 1996). Con su concepción narrativista del discurso histórico, Vicetto será la única excepción.

Otro aspecto común a todos estos autores del s. XIX, es su preocupación por el carácter educacional y divulgativo que sus obras debían tener. No se trataba tan sólo de enfrentarse a un público erudito, sino de divulgar a todos los niveles sociales que la realidad histórica de la identidad del pueblo gallego había existido desde la más remota Prehistoria. Con este fin, gran número de conferencias sobre historia y prehistoria se leen en los Juegos Florales de Vigo, de Ferrol... junto a obras políticamente importantes, como las de Alfredo Brañas (1889 y 1889a) o Macías y García (1892). Lengua gallega, historia gallega, política gallega, se entremezclan en estos actos de exaltación y difusión de la identidad y la nación gallega. Manuel Martínez Murguía fue siempre un asiduo participante y promotor de este tipo de actividades (1888, prólogo; Máiz Suárez 1984 y 1997); y todas las demás Historias de Galicia de los autores gallegos que escriben en el s. XIX responden al fin predeterminado de dar al pueblo gallego una conciencia histórica de su identidad cultural y nacional, de la que hasta el momento no se había dado cuenta y que por tanto permanecía dormida en su seno.

Todos estos procesos se encuentran estructuralmente integrados en una intensa vida cultural, con un afán dinamizador y de divulgación de la identidad gallega. No sólo en actos públicos se sustentaba este afán, sino que alcanzó a la prensa, sobre todo a la prensa local (Murguía 1888, prólogo; Villares Paz, R., 1984/85). El concepto de Historia de los autores 
del s. XIX imponía la necesidad práctica de la divulgación de ciertas «verdades históricas», entre ellas la veracidad pre-histórica de la identidad gallega. Hasta estos momentos, estas cuestiones eran de orden intelectual, es decir, propias de una elite que tenía acceso a la cultura. La gran masa social gallega, el campesinado, permanecía ausente de estas cuestiones, ya que el grado de alfabetización era casi nulo. El problema era hacer ver al pueblo gallego que era diferente al resto de España, que era mejor porque sus raíces eran las más antiguas y su raza la más ilustre. Los historiadores del s. XIX establecen por primera vez una conexión directa entre el campesino (base de la sociedad gallega) y las elites intelectuales a través de la Historia, mediante la demostración de las pervivencias históricas de un pasado celta en el campesinado actual gallego. Esta relación directa entre pueblo gallego y su origen prehistórico y celta a través del campesino se convirtió en un argumento fácil de asumir por todas las clases sociales, sobre todo por las más bajas y menos alfabetizadas.

El celtismo como pervivencia folklórica y racial es un argumento que permanece asumido casi inconscientemente por la mayor parte de la población gallega e incluso por alguna parte de sus investigadores (B. García Fernández-Albalat 1997), a pesar de las controversias intelectuales y académicas que todavía está planteando (Fernández-Posse 1998). El celtismo del Noroeste Peninsular es, para gran parte de los investigadores contemporáneos, una reminiscencia de teorías raciales, más que un tema paleoetnológico. El concepto de «celta» $\mathrm{o}$ «céltico» lleva el poso de esta carga ideológica que los autores del XIX le asignaron. Separar el término histórico y paleoetnológico de las cargas ideológicas actuales es un proceso necesario de deconstrucción del término (Ruíz Zapatero 1993, 2001), hasta vaciarlo de contenido ideológico.

\section{EL SEMINARIO DE ESTUDOS GALEGOS}

El Seminario de Estudos Galegos institucionaliza, con su creación en 1923, el saber histórico en todo lo relacionado con la región gallega. Toda la actividad cultural e intelectual, que antes era sobrellevada por los que Martínez Murguía denominó los «precursores» (1886), queda institucionalizada y regulada a través del Seminario. Los trabajos de esta institución son publicados a través de la Revista Nós, asociada a su vez a la 
Xeración Nós. Es decir, lo que antes eran aportaciones individuales de eruditos y aficionados, se canalizan a través de un Seminario cuya voz pública y medio de divulgación es una publicación cuyas orientaciones regionalistas eran evidentes. El positivismo de los historiadores es tan evidente que ahora todas las alusiones a la intención práctica de elaborar una identidad nacional a partir de sus obras desaparecen, eludiendo cualquier alusión al subjetivismo en el proceso de investigación. La Historia se concibe, desde la aparición de Seminario, como una verdad objetiva, carente de una funcionalidad social y política. Se abandonan los ideales románticos sobre el carácter práctico de la Historia y, sobre todo, el carácter divulgativo popular que había orientado la labor de estos primeros historiadores. La especialización científica de la Arqueología y la Prehistoria empiezan a resultar en un cuerpo profesional que demanda una literatura específica, especializada, e inaccesible tanto física como intelectualmente a la gran masa de la población. Comienza un proceso de escisión entre la comunidad que investiga, que escribe la Historia, y las capas con menor acceso a la educación de la población, que tendrá su máxima expresión en el presente. El conocimiento histórico empieza ahora a circular en los cerrados niveles del mundo intelectual y académico, y el pueblo se queda con la visión del celtismo que los precursores y durante algún tiempo más la Cova Céltica irán difundiendo. Esto dará lugar a que, incluso hoy, exista una visión popular del celtismo, que responde a la visión del s. XIX, y una visión académica del mismo (en realidad, varias visiones dialécticas, como veremos), y que ambas esferas permanezcan prácticamente impermeables la una a la otra.

Como mencioné más arriba, no existe incoherencia entre la elaboración e interpretaciones históricas y la ideología de los autores. El particularismo histórico y los estudios raciales se desarrollaron, ligados normalmente al celtismo, pero desprovistos parcialmente de la profunda carga práctica que los «precursores» explicitaban en sus obras. Aunque invisible, esa carga existía ahora ocasionalmente en el marco de estudio (Galicia), y el positivismo como modelo teórico imperante (Hernando 1992; Trigger 1992), además de permanecer sobre todo en la ideología y la filosofía de la institución que la publicaba. El celtismo empezaba a flaquear sobre todo por las críticas a su uso político que se hacían desde Madrid y por la incipiente decadencia de las teorías y metodologías raciales (Pereira González 1997; Eriksen 1993). El Galleguismo necesitaba de algo menos 
criticable que la asunción de la pertenencia a una raza desde tiempos prehistóricos.

El uso de los datos arqueológicos, ya muy evolucionado (Pereira Gonzalez 1996), hizo que las metodologías del siglo pasado se superasen a través de un positivismo de carácter más normativista. La Historia ya no era un bloque homogéneo, sino que, ya desde Villa-amil y Martínez Padin (Pereira González, 1997) empezó a subdividirse. Existían etapas concretas con características concretas, tanto espacial como cronológicamente, que estaban definidas a través de una cultura material concreta. Galicia se sumó a la corriente teórica de la época, y adoptó una metodología más descriptiva para estudiar el pasado. Además, la cultura material de las etapas prehistóricas y protohistóricas gallegas tiene para muchos una idiosincrasia particular, que la diferencia del resto de las culturas peninsulares, ahora materialmente además de históricamente. Pero sigue siendo el pasado de Galicia, y siguen buscándose líneas que unan ese pasado con el presente. El marco geográfico y la existencia de una misma identidad geográficamente definible en el presente y en la prehistoria son algunas. Las tradiciones folklóricas de V. Risco o los rasgos culturales que la particular fisionomía del territorio gallego impone son otras. Cuevillas se convierte en el principal impulsor del celtismo en Galicia. Pero su trabajo no se puede entender separado de los trabajos de Geografía, Etnografía o Historia que el Seminario (1934) apoya y subvenciona, y que la Revista Nós se encarga de divulgar entre los intelectuales gallegos y quizá en menor medida hacia el pueblo.

Como dice Fernández-Posse, el celtismo terminó de arraigar en estas capas populares de Galicia convirtiéndose en un símbolo de la nacionalidad y de la identidad gallega. De esta labor de afianzamiento del celtismo se encargaba un aparato intelectual, el Seminario de Estudos Galegos, que daba una cohesión y una determinada orientación galleguista a las investigaciones que se elaboraban, y un apoyo institucional como medio de divulgación, la Revista Nós, que se encontraba profundamente vinculada a la vida cultural de la época.

El Seminario da un cuerpo institucional a la investigación histórica. La Historia que se hace con él ya no se considera un instrumento, sino que empieza a considerarse políticamente aséptica. Esto, sumado a la aparente objetividad de los modelos teóricos positivistas de la época, y al soporte académico (ya que la Arqueología empieza a reconocerse en la

«CUADERNOS DE ESTUDIOS GALLEGOS», Tomo XLVIII, Fascículo 114, Santiago 2001. 
Universidad) hace que la intención galleguista o regionalista se traslade de la Historia a la Institución. La Historia que se hace en el Seminario de Estudos Galegos es objetiva, pero sigue demostrando, ahora con mayor cientificidad que en el s. XIX, que Galicia tiene una serie de características que la han diferenciado de Castilla y España, que como afirman Rojo Salgado (1989), Máiz Suárez (1984, 1997) y Renales Cortés (1996) es siempre el elemento diferenciador por oposición a lo gallego durante toda la Historia. El Seminario, al orientar todas las investigacionęs hacia el territorio gallego y sus características, toma el relevo del regionalismo del XIX. Porque siguen presentes los mismos temas: Galicia, sus características diferenciales en cualquier aspecto de la Ciencia. La única diferencia con la Historia que se hacía en el s. XIX es el cambio de modelo teórico: del narrativismo y el historicismo clásico al predominio de los Círculos Culturales. El auge del conocimiento arqueológico fue dando tintes de normativismo a numerosas investigaciones, manteniéndose como centro de estudio lo que interesa al Galleguismo.

\section{LOS TIEMPOS DIFÍCILES}

El Instituto Padre Sarmiento de Estudios Gallegos, dependiente del Consejo Superior de Investigaciones Científicas, encabeza el primer volumen de sus Cuadernos de Estudios Gallegos (1944/45) con el retrato ecuestre del Generalísimo Franco, pintado por Sotomayor. La política vuelve a imponerse sobre el conocimiento histórico. En su introducción se declara que lo que se intenta es recoger todos aquellos trabajos e investigaciones que han quedado sin rumbo desde 1936. Algunas obras que habían sido comenzadas por el Seminario de Estudos Galegos son retomadas, como la elaboración de Bibliografías actualizadas y generales sobre Galicia, cuya última actualización fue en 1976/77.

Es un lugar común en la historiografía gallega la afirmación de que los Cuadernos de Estudios Gallegos, afines al régimen y sometidos a una censura impenetrable, se ven incapacitados para continuar libremente con la labor del Seminario (Acuña 1995; Armada Pita 1999; Díaz-Andreu 1997, 1997a; Díaz-Andreu y Mora 1995; Fernández-Posse 1998; González Reboredo 1990; Pereira González 1996, 1997). Esto es así a la hora de afirmar una identidad cultural y nacional gallega abiertamente. Sin em- 
bargo, tras realizar un vaciado bibliográfico de los Cuadernos (Díaz Santana, inédito ${ }^{2}$ y 2002), no es sorprendente ver que las analogías etnográficas entre el campesinado actual y las comunidades prehistóricas, aún tenidas por célticas por muchos de estos autores incluso a mediados de los cincuenta (Cuevillas 1956), siguen siendo publicadas. No sólo continúan apareciendo en los Cuadernos obras de Cuevillas, que se convierte en el epígono de la visión más clásica del celtismo, sino que incluso autores españoles publican visiones particulares sobre el carácter celta de la cultura material de la Edad del Hierro del NO. Por ejemplo, en 1946/47 García y Bellido publica un pequeño artículo que establece analogías entre algunas viviendas campesinas gallegas actuales y las prehistóricas, sin establecer la permanencia de la misma identidad cultural, pero dando por supuesto que la cultura es similar, tema que ya había mantenido en su publicación con Obermaier (1932); en el mismo número Jorge Días defiende un celtismo a ultranza en cuanto a las construcciones circulares. Aunque en el título de su artículo no habla de Galicia, durante su desarrollo sigue los mismo argumentos del s. XIX: cultura celta que pervive en las comunidades campesinas actuales. Es significativo que la palabra «Galicia» desaparece de los títulos de las colaboraciones, siendo sustituida por «Noroeste» hispánico o de la Península Ibérica, mucho más aséptico y menos comprometedor a la hora de la superar la censura, e históricamente más correcto. Sin embargo, el contenido de algunos de estos artículos sigue aludiendo al celtismo, y a ciertas pervivencias en la cultura material campesina contemporánea de algunos elementos celtas prehistóricos, como por ejemplo la arquitectura castreña (teoría que parece seguir vigente, a tenor de lo publicado en la revista National Geographic, Vol. X, no 4, Abril de 2002). Pero esto no suponía en todos los casos la pervivencia de las connotaciones nacionalistas que el concepto celta había adquirido en el s. XIX. El celtismo como interpretación empezaba a intentar deshacerse de su marbete nacionalista. Esta aceptación del celtismo como interpretación estrictamente histórica ha sido recientemente demostrada en la producción de Carlos Alonso del Real (Armada 1999), tradicionalmente considerado el ejemplo más claro de anti-celtismo.

${ }^{2}$ DÍAZ SANTANA, B. (inédito): Arqueología y Política en Galicia: una Revisión Historiográfica. Memoria de Licenciatura Presentada en el Departamento de Prehistoria de la U.C.M. Noviembre de 1999.

«CUADERNOS DE ESTUDIOS GALLEGOS», Tomo XLVIII, Fascículo 114, Santiago 2001. 
La Edad del Hierro, no bien delimitada cronológicamente, siguió siendo el período que más atención recibía. Los límites geográfícos, como en el s. XIX, siguieron siendo los de la región gallega. La última publicación que defienda abiertamente el celtismo en Galicia, parece ser la de Luengo Martínez (1971). De entonces en adelante el celtismo desaparece de los Cuadernos, aunque pervive en otras publicaciones (Bermejo 1978).

Otro tema que se sigue tratando es el estudio de la relación del Noroeste Peninsular con el resto de comunidades atlánticas, como Bretaña, Gran Bretaña y sobre todo Portugal, ya sea a través del comercio (Cuevillas 1949), de la analogía de sus construcciones (Cuevillas y Lorenzo 1946/ 47; Jorge Días 1946) o de cualquier aspecto de la cultura material (Cuevillas 1950a; Bouza Brey 1950).

Las cuestiones sobre etnicidad siguen vigentes en todos estos trabajos que se publican en los Cuadernos de Estudios Gallegos justo después de la Guerra Civil. Bajo títulos bastante asépticos, siguen tratándose los viejos temas, aunque la cuestión de la identidad cultural y nacional del pueblo gallego no se trata explícitamente. Está inmersa en la propia realidad de tratar de una historia propia de Galicia y del pueblo gallego, en determinados estudios que no siempre adoptan interpretaciones desde el celtismo.Los Cuadernos de Estudios Gallegos no acaban tan radicalmente como se ha supuesto con la vida cultural gallega, ni suponen un retroceso en la línea de investigación que se venía haciendo desde el s. XIX, al contrario de lo que afirman la mayoría de los autores. Sino que los autores gallegos y galleguistas superan la censura eliminando la asociación explícita entre pasado y presente. En mi opinión, la vinculación nacionalismo = celtismo se enfatizó desde la propia producción historiográfica gallega, ya que al eliminar a posteriori el celtismo como interpretación se pretendía eliminar también toda posible acusación de nacionalismo por parte del resto de la comunidad investigadora peninsular. Pero el poso dejado en el s. XIX otorgó a la historia y la prehistoria de Galicia un carácter gallleguista y autóctono, de lo galaico frente a lo castellano, incluso en la propia idiosincrasia galaica de la cultura material del NO. tan defendida por los investigadores más normativistas incluso en la actualidad $^{3}$. Que el pueblo gallego es distinto es algo que está implícito

${ }^{3}$ Agradezco esta observación al Dr. César Parcero. 
en el objeto de su investigación, ya que es la asunción apriorística de esta diferencia la que orienta tanto el objeto de estudio como sus conclusiones. Esta identificación radical del concepto celta con uno de sus significados, el celtismo como ideología nacionalista, y la reivindicación de una mayor veracidad y objetividad mediante su rechazo explícito permanece en algunos sectores de la producción historiográfica gallega contemporánea sobre la Cultura Castreña (de la Peña 1997: 144).

En torno a 1968, comienza a ser más importante la publicación por los Cuadernos de colaboraciones escritas en lengua gallega, en cualquiera de los temas de los que trata, que se mantienen siempre bajo el tema común de Galicia y lo que le es propio y característico (Carballo Calero 1968; García Martínez 1968; Acuña Castroviejo 1971, 1980; Arias Vilas 1971; Romero Masia 1984/85; Villares Paz 1984/85). Al mismo tiempo el celtismo y las alusiones a la realidad paleoetnológica del Noroeste peninsular van desapareciendo. ¿Por qué, cuando el objeto de investigación es el mismo: la cultura castreña o Edad del Hierro? El celtismo había constituido la principal arma ideológica del galleguismo del s. XIX y de los primeros años del s. XX (del Seminario de Estudos Galegos, la famosa Cova Céltica). Como tal, no sólo fue atacado por la censura, sino que las alusiones al celtismo, aún presentes en los Cuadernos, fueron perdiendo credibilidad. Este proceso ya se había iniciado en el s. XIX, cuando desde Madrid se criticaba el celtismo de ciertos autores galleguistas (los precursores). Daba igual el modelo teórico o interpretativo, o la orientación ideológica real del trabajo. En un proceso metonímico, el galleguismo se asoció al celtismo, y a medida que tras la II Guerra Mundial las nuevas teorías normativistas empezaron a sustituir definitivamente al historicismo y los modelos histórico culturales, quedó un vacío que era necesario cubrir metodológicamente. Los autores y autoras gallegos dejaron de escribir sobre Galicia, para hacerlo sobre el Noroeste Peninsular, pero conservaron a grandes rasgos los límites geográficos anteriores. La cronología fue afinada a través de métodos de datación más exactos, y la realidad material sustituyó a las definiciones étnicas. Los datos materiales propios de culturas pasadas sobre nuestro suelo escribieron la letra de la nueva historia de Galicia, y constituyeron sus más viejas raíces.

Pero no sólo había que sustituir al celtismo como elemento identificador de la identidad gallega, sino que se convirtió en un anatema propio de la investigación de los autores gallegos. El recuerdo de la ecuación «celtis- 
mo -nacionalismo- teorías no científicas» constituyó un desprestigio para la investigación, como muy bien expresa A. de la Peña cuando afirma que éste se convirtió en una lacra historiográfica (1992 y 1995). Había que luchar contra la asunción por parte de la vida académica centralista de que la investigación que se realizaba en Galicia era poco científica ya que estaba orientada hacia el nacionalismo, y en mi opinión esto se hizo rechazando desde dentro la manifestación más característica del mito nacionalista: el celtismo.

Desde mediados de los setenta el celtismo que aún pervivía en algunos autores desde una nueva perspectiva antropológica (Bermejo 1978) se sustituyó por un autoctonismo más que exacerbado, con un interés desmedido por establecer unos límites geográficos casi matemáticos para la cultura castreña, interés que aún permanece en el presente (Calo 1997; de la Peña 1992, 1995, 1997; de la Peña y Vázquez 1996; Esparza 1993; Pereira Menaut 1997; Vázquez y Cano 1997). En estos momentos las preocupaciones de los arqueólogos son la descripción y la clasificación tipológica. El Normativismo es lo que define lo galaico, a través del estudio de la cultura material sin afán interpretativo. Eso sí, siempre una cultura material con su propia idiosincrasia, netamente galaica y por tanto, como reivindica Calo (1993 y 1997), aprehensible únicamente a través del idioma autonómico actual. Dentro de esta postura teórica, la definición del concepto de etnia y su adscripción a culturas pasadas es erradicado de la literatura científica porque se considera inalcanzable. Lo mismo sucede cuando se adoptan metodologías procesuales. $\mathrm{Al}$ igual que en el resto de Europa, los intentos por cualquier historiador y arqueólogo de aproximarse al estudio de las etnias del pasado, presuponía una ecuación directa entre cultura material y etnia, o pueblo y raza, con todas las fatales consecuencias que ello supuso durante la Segunda Guerra Mundial. Este sentido peyorativo del término etnia, que se asociaba conceptualmente al de raza y al de genocidio, era paralelo al de celta, que se asociaba a su vez con galleguismo y nacionalismo. El proceso ha sido similar en toda Europa, y aún podemos ver restos de esta asociación en autores actuales, tanto europeos (Collis 1996, 1996a, 1997; James 1998, 1999; Renfrew 1996) como gallegos (Calo 1993, 1997; de la Peña, 1992, 1995, 1997; Pereira Menaut 1994). Son sobre todo los autores gallegos los que rechazan los estudios etnológicos, mientras otros autores peninsulares, M. AlmagroGorbea, A. Lorrio, G. Ruiz Zapatero, A. Jimeno o F. Marco Simón, en la 
actualidad, estudian aspectos del mundo celta desde fuera de Galicia. Lo celta, entendido desde el poso histórico de la historiografía gallega, ha perdido toda credibilidad científica, y se ha convertido en un término manipulado política y culturalmente y por tanto carente para muchos de validez histórica, como parece haber sucedido en otras partes de Europa.

\section{VINO VIEJO EN ODRES NUEVOS}

Sin embargo el celtismo (como algo más que el hecho lingüístico) ha resurgido en los últimos años en algunas investigaciones gallegas y europeas al mismo tiempo que el nacionalismo lo ha hecho en la política gallega, española y europea. Algunos de los investigadores gallegos han retomado el estudio de lo celta desde la Arqueología (Arias Vilas, 1997; Brañas 1995; García Quintela 1996; García Fernández-Albalat 1997; Pena 1993, 1994; Parcero Oubiña 1997). Esto explica el surgimiento en los últimos años de dos corrientes de celtismo en Galicia. La primera de ellas es la que tiene como objeto de estudio la cultura celta prerromana, es decir, la que entiende que existe una homogeneidad cultural en la Europa Atlántica, relacionable con el mundo Celta que describen las fuentes clásicas y que ha dejado reminiscencias en la Edad Media. En este sentido, es similar a los estudios del mundo celtibérico (Burillo 1998) o céltico peninsular (Almagro 1993, 1993a, 1994). La otra versión del celtismo gallego actual es la que retoma la línea del galleguismo, y convierte lo celta en un elemento de identidad cultural actual que pervive desde la prehistoria. Se podría afirmar que es el resultado del desarrollo del celtismo en la mentalidad popular, aunque también se mantiene desde algunas investigaciones. Fernández-Posse (1998) afirma que el celtismo permanece en Galicia únicamente como reminiscencias decimonónicas, convertido un cajón de sastre donde caben todas las características que definen lo gallego y lo atlántico, como también dice J. Collis (1996): música celta, arte celta... un conglomerado de rasgos culturales prerromanos, medievales y modernos que se transforman en un nuevo concepto de celta (Ruíz Zapatero 2001). Este concepto ha sido creado en la actualidad por un colectivo social que desea fundamentar históricamente su propia identidad. Como en el s. XIX, lo celta se retoma como representación de una identidad cultural y/o nacional. Este sentido del concepto celta, so- 
cialménte hablando, ha sido creado culturalmente en el presente, y sólo se puede entender y explicar desde este punto de vista. No es posible asociarlo al concepto de celta que se maneja dentro de la historiografía, ya que aquél es una creación cultural actual, y éste es la aplicación de un término histórico, por cuanto responde, según los escritores clásicos, a una realidad histórica prerromana. El problema es que ambos conceptos se entremezclan a menudo, pues ambos usan como medio de comunicación los mismos recursos. El ostracismo académico, raramente superado (Pena 1993), obliga a todo el que quiera publicar algo a entrar en el mundo social de lo celta. Así, congresos como los de Ferrol, con títulos ambiguos como Os Celtas da Europa Atlántica. II Congreso Internacional sobre a Cultura Celta, entremezclados con festivales náuticos de las comunidades atlánticas, célticas, junto a los cuales permanecen representaciones de asociaciones nacionalistas con la Liga Céltiga Galaica; o ediciones de música celta junto con trabajos sobre la «Cultura Celta de los Castros», como el de Felipe Arias, para C.O.D.A. Music S.A. (Edita Sons de Galiza). Lo sociológico y contemporáneo se entremezcla con lo histórico, obligado por un mundo universitario reacio a todo lo que recuerde a la vieja Cova Céltica, de la que paradójicamente está bastante orgulloso por sus ideales políticos. Es la misma dinámica cultural del s. XIX, salvo que los Juegos Florales se han convertido en Congresos y Festivales, y en la actualidad esta identidad gallega se reivindica desde el celtismo y desde la celtofobia. De este modo, es imposible que el estudio de lo celta como interpretación histórica supere la lacra que supuso su manipulación en el s. XIX, cuando usa los mismos medios que entonces y al mismo tiempo debe superar los prejuicios frente a todo lo celta de la investigación gallega y española.

Existe en la actualidad otra línea de galleguismo que utiliza como definición lo galaico prerromano o los primeros momentos de la ocupación romana, y la negación de todo elemento celta. El autoctonismo de autores como de la Peña, Calo Lourido o Pereira Menaut expresa con diferentes palabras el mismo deseo de resaltar lo galaico frente a lo español. Dentro de un discurso que utiliza el pasado en el proceso de creación de la identidad gallega, lo galaico, tradicionalmente aislado del resto de la Península Ibérica y de la Europa Atlántica, como demuestra para estos autores el autoctonismo del proceso de formación de la Cultura Castreña (en el caso de Calo 1993 y 1997, y de la Peña 1997), o la idiosincrasia de la organiza-

«CUADERNOS DE ESTUDIOS GALLEGOS», Tomo XLVIII, Fascículo 114, Santiago 2001. 
ción social castreña en la romanización (en el caso de Pereira Menaut 1997), se convierten junto con la idiosincrasia de la cultura material protohistórica en un hecho diferencial galaico, y por extensión gallego, a través de la historia. Los celtas han sido sustituidos por galaicos, tal como ha señalado M. D. Fernández-Posse (1998), pero estos siguen siendo los primeros gallegos del Noroeste de la Península Ibérica.

El celtismo como corriente interpretativa en las investigaciones protohistóricas gallegas sigue asociado a una vida social que utiliza el concepto celta como término de identidad gallega. El escaso apoyo institucional (tanto político como universitario) y por tanto bibliográfico (revistas, editoriales...) obliga a estos autores a vincularse, aunque sea sólo nominalmente, a este movimiento ideológico. El concepto histórico de celta sigue asociado al político y sociológico a través de la vida cultural gallega. Algunas editoriales, como A Nosa Terra, mantienen la misma filosofía que en su tiempo tuvo el Seminario de Estudos Galegos: con una vinculación nacionalista explícita, edita y subvenciona investigaciones de autores celtistas y anticeltistas (A Nosa Historia, 6, Ed. A Nosa Terra; Calo 1993 y 1997). El sentido de esta contradicción es buscar una dialéctica propia a Galicia, independiente de las españolas, las peninsulares e incluso las europeas: las fronteras culturales de los estudios que se editan no rebasan las de la Comunidad Autónoma.

La Universidad se sostiene, en cuanto a soporte bibliográfico, a sí misma. Un examen de las publicaciones (Gallaecia, Cuadernos de Estudios Gallegos) sigue demostrando la ausencia del celtismo como interpretación, superada en parte por un resurgir de los modelos trifuncionales de Dumézil (Parcero 1995, 1997). Pero en este sentido, lo indoeuropeo, menos manipulado políticamente en Galicia, tiene más aceptación que lo celta, cuya manipulación ha hecho de esta palabra algo inservible (Parcero 1997: 38).

\section{CONCLUSIONES}

A lo largo de la producción historiográfica gallega, el concepto de Historia, entendido como el proceso de investigación y de elaboración historiográfica del hecho histórico, ha ido asumiendo diferentes connotaciones y acepciones. Sin entrar a discutir aspectos epistemológicos del 
concepto, he pretendido abordar un estudio diacrónico del mismo. El carácter de la Historia dentro de la vida social de la época de cada autor ha cambiado radicalmente desde el s. XIX hasta el presente. La producción Historiográfica gallega asumía conscientemente que la Historia tenía una razón de ser sobre todo práctica. Se ha asumido que, en estos primeros momentos de la historiografía, la Historia era una ocupación de las clases más altas de la sociedad (Jensen 1997). Esto es así en el caso gallego, ya que los primeros en elaborar Historias de Galicia pertenecían sobre todo a clases burguesas, y constituían una elite intelectual. Aquí resalta la asunción explícita del carácter sobre todo divulgativo de su labor. A través de actividades sociales en las que estos autores participaban, se pretendía extender el conocimiento histórico, o parte de él, entre toda la masa social. Esto respondía al objetivo de concienciar a todo el pueblo gallego, independientemente de su clase social, de la existencia de una identidad gallega. Esta identidad, en el s. XIX, se fundamentó en la existencia de continuidades raciales, etnográficas y culturales, es decir, en la existencia de una misma identidad desde la prehistoria, en concreto desde la Cultura Castreña. El carácter celta de la Cultura Castreña, y la pervivencia del carácter celta de la identidad gallega a lo largo de la Historia constituyeron su mito nacionalista.

La Historia como conocimiento fue institucionalizada en Galicia con el Seminario de Estudos Galegos, que dio una homogeneidad ideológica y filosófica a los estudios llevados a cabo por sus miembros y una difusión del conocimiento sobre la Cultura Gallega a través de la Revista Nós. En Galicia como en el resto de España la institucionalización del conocimiento histórico y la profesionalización de la Arqueología y la Historia llevaron consigo una escisión entre éste y la masa social. En el caso gallego, la divulgación del conocimiento histórico se seguía produciendo a través del Seminario, pero en cuanto al concepto de Historia, ya no tenía el sentido práctico del s. XIX, su finalidad ya no era justificar la nación gallega. El conocimiento histórico se entendía como una verdad objetiva, alcanzable a través de la investigación, y sin connotaciones de tipo subjetivo o práctico alguno. Todo aquello demostrado por la Historia se consideraba una verdad científica irrefutable. La orientación ideológica se trasladó del concepto de Historia como herramienta política al de la institución que aglutinaba y coordinaba los estudios realizados, en este caso el Seminario. La transmisión de este conocimiento a todas las clases sociales 
dejó de ser el objetivo primordial de la Historia. Esta tendencia ha continuado hasta el presente, en que los resultados de la investigación se mueven entre círculos especializados (el mundo académico, publicaciones especializadas) y se ha abandonado todo intento de difusión popular.

El celtismo como teoría histórica y política es un buen ejemplo de esto. El afán dinamizador y de difusión cultural del s. XIX universalizó en el pueblo gallego, o por lo menos lo intentó con las capas más bajas, la existencia de una realidad celta en la Prehistoria cuya identidad habría permanecido inmutable hasta el presente. En estos primeros momentos, el celtismo como teoría estrictamente histórica (como en el resto de Europa) estaba estructuralmente integrado en un concepto de Historia instrumental .Esta identidad históricamente demostrada, sería la esencia de la identidad gallega, sobre todo en el mundo campesino, y el objetivo era difundir la existencia de la identidad gallega, junto con una explicación histórica de su origen. El conocimiento histórico, salvo en el caso de Vicetto, se entendía de una manera positivista. Es decir, las interpretaciones históricas se consideraban verdades absolutas. Ahora bien, era el concepto en sí de Historia, y no las interpretaciones realizadas, el que llevaba en sí el poso nacionalista. Cualquiera que fuese la interpretación histórica ésta significaba en todo momento que era una historia exclusivamente gallega y anticentralista. En el caso gallego, el celtismo como interpretación respondió a este concepto de Historia. Y por ejemplo, en el caso portugués, el anticeltismo de F. Martíns Sarmento respondió a la creación de su identidad contemporánea tanto desde el celtismo, en un primer momento, como desde el anticeltismo en sus últimos trabajos (González-Pardo 1983).

Sin embargo, la orientación ideológica nacionalista o galleguista se identificó totalmente con el celtismo, de manera que su rechazo supuso una mayor consideración hacia las investigaciones realizadas por autores gallegos por la comunidad científica. Este proceso ha continuado hasta el presente. El rechazo del celtismo no ha invalidado las connotaciones ideológicas de la disciplina, y de sus investigaciones. En este sentido, mantengo la postura marxista de la historiografía de O. W. Jensen (1997), y asumida por la mayoría de los autores para la historiografía del s. XIX, definiendo la Arqueología y/o la Prehistoria como una labor sobre todo de la clase burguesa cuyo principal objetivo es la justificación de su identidad. En el caso gallego, esto se lleva a cabo en el presente desde diversas teorías interpretativas, como son el celtismo, el autoctonismo o el

«CUADERNOS DE ESTUDIOS GALLEGOS», Tomo XLVIII, Fascículo 114, Santiago 2001. 
normativismo. La coherencia entre estos modelos teóricos y metodológicos y la orientación identitaria galleguista se produce en el momento en que se identifica un rasgo del pasado - ya sea lo celta, la idiosincrasia de la cultura material, social, etc. - y se reifica en el presente convirtiéndolo en un elemento histórico de la identidad gallega actual. Sin embargo, la escisión entre la comunidad investigadora y el resto de la población a través de la especialización y la profesionalización, y la absoluta impermeabilidad entre ambas en el presente, han evitado la difusión de las controversias y dialécticas historiográficas en torno al tema del celtismo.

\section{BIBLIOGRAFÍA}

AA. VV: (1944-1945): Cuadernos de Estudos Galegos, Vol. I, Santiago de Compostela.

AA.VV. (1920-25): Nós, Boletín Mensual da Cultura Galega, T. I, nº 1 24, Ed. Galaxia, A Cruña.

AA.VV. (1934): Seminario de Estudos Galegos. Dez cursos de traballo: 1923-1934, Compostela.

ACUÑA CASTROVIEJO, F. (1995): «Historiografía e investigación de la Cultura Castrexa en Galicia», en J.M. HIDALGO CUÑARRO (coord.), A Cultura Castrexa galega a debate, Actas del Curso de Verano de la Universidad de Vigo, 1995, Instituto de Estudos Tudenses: 25-39.

ACUÑA CASTROVIEJO, F. (1971): «Catro machados de bronce inéditos», C.E.G., n ${ }^{\circ} 26$, fasc. $78: 42-44$.

ACUÑA CASTROVIEJO, F. (1980): «A Arqueoloxía na obra de López Ferreiro», C.E.G., $\mathrm{n}^{\circ}$ 32, fasc. 96-97: 57-80.

ALMAGRO-GORBEA, M. (1993): «Secuencia cultural y etnogénesis del centro y noroeste de la Península Ibérica», en Actas del XXII Congreso Nacional de Arqueología, 1993, Vol. I, Vigo:121-138.

«CUADERNOS DE ESTUDIOS GALLEGOS», Tomo XLVIII, Fascículo 114, Santiago 2001. 
ALMAGRO-GORBEA, M. (1993a): «Los Celtas en la Península Ibérica: Origen y Personalidad Cultural», en M. ALMAGRO-GORBEA y G. RUÍZ ZAPATERO (eds.). Los Celtas: Hispania y Europa, Editorial Actas, Madrid: 121-172.

ALMAGRO-GORBEA, M. (1994): «El urbanismo en la Hispania 'Céltica': castros y oppida», en M. ALMAGRO-GORBEA y A. M. MARTÍN BRAVO (eds.), Castros y Oppida en Extremadura. Editorial Complutense, Complutum Extra 4: 13-76.

ALONSO TRONCOSO, V. (1995): «La Cultura Castreña en Galicia: historiografía arqueológica de los últimos años (1980-1996)», en CuPaUAM, 22: 101-124.

ARIAS VILAS, F. (1997): «Pervivencias e transformacións na sociedade galaico-romana», en Sociedade e Categorías sociais na Historia de Galiza,Vigo, Ed. A Nosa Terra, Col. A Nosa Historia, 6, 1997: 18-22.

ARIAS VILAS, F. (1998): «Trabajo sobre la Cultura de los Castros», CD-Rom de Música Celta, Edita Sons de Galiza (C.O.D.A. Music s.1.).

ARIAS VILAS, F.; DURÁN FUENTES. M. C. (1996): «A Cultura Castrexa», C.R.O.A., 6, e-castrexo.es: 31-37.

BARREIRO FERNÁNDEZ, X.R. (1993): «A Historia da Historia. Aproximación á Historiografía Galega: de Murguía a Risco (siglos XVI XIX)», en J. GARCÍA BERAMENDI (coord.), Galicia e a Historiografia, 1993, La Coruña, Tórculo Ediciones: 183-209.

BARREIRO FERNÁNDEZ, X.R. (1988): «La historia de la Historiografía gallega (siglos XVI-XIX), en IV Jornadas de Historia de Galicia, Historiografia gallega, 1988, Orense: 15-80.

BARROS, C. (1994): «Mitos de la Historiografía Galleguista», Manuscrits, $\mathrm{n}^{\circ} 12$, Gener: 245-266.

BATE, L. F. (1998): El proceso de investigación en Arqueología, Barcelona, Ed. Crítica.

«CUADERNOS DE ESTUDIOS GALLEGOS», Tomo XLVIII, Fascículo 114, Santiago 2001. 
BERMEJO BARRERA, J.C. (1998): «La Historia como sistema de comunicación», en Gallaecia, 17, Santiago: 351-370.

BERMEJO BARRERA, J.C. (1989): «Los antepasados imaginarios en la historiografía gallega», C.E.G., T.XXXVIII, fasc.103, Santiago 1990: 73-91.

BERMEJO BARRERA, J.C. (1993): «Sobre la construcción del objeto historiográfico. Consideraciones sobre el método de la Historia de la Historiografía», en J. GARCÍA BERAMENDI (coord.), Galicia e a Historiografia, 1993, La Coruña, Tórculo Ediciones: 7-28.

BERMEJO BARRERA, J.C. (1978): La Sociedad en la Galicia Castreña, Santiago de Compostela, Ed. Follas Novas.

BRAÑAS MENÉNDEZ, A. (1889): El Regionalismo. Estudio sociológico, histórico y literario, Ed. Jai-Molinas, Barcelona, 1889, $1^{\text {a }}$ edición, en Obras Selectas, Ed. Xuntanza.

BRAÑAS MENÉNDEZ, A. (1892): La crisis económica en la época presente y la descentralización regional, Imprenta de J. M. Paredes, Santiago de Compostela, Santiago, 1892, $1^{\text {a }}$ edición, en Obras Selectas, Ed. Xuntanza.

BRAÑAS, R. (1995): Indíxenas e romanos na Galicia Céltica, Librería Follas Novas, Santiago de Compostela, 1995.

BURILLO MOZOTA, F. (1993): «Aproximación a la Arqueología de los Celtíberos», en M. ALMAGRO-GORBEA \& G. RUÍZ ZAPATERO (eds.), Los Celtas: Hispania y Europa, Ed. Actas, Madrid: 223-253.

BURILLO MOZOTA, F. (1998): Los Celtíberos. Etnias y Estados. Editorial Crítica, Barcelona 1998.

CALO LOURIDO, F. (1994): «Manifestacións da plástica castrexa na provincia de Lugo», C.R.O.A., 4, e-castrexo.es:-10-11. 
CALO LOURIDO, F. (1997): A Cultura Castrexa, Edicións A Nosa Terra, $2^{\mathrm{a}}$ edición ( $1^{\mathrm{a}}$ edición en 1993), Colección Historia de Galicia 3, Vigo, $222 \mathrm{pp}$.

CARTELLE FERNÁNDEZ, C. (1998): «Benito Vicetto na historiografía galega», en V. ALONSO TRONCOSO (ed.), Ferrolterra Galaico-romana, Ed. Exmo. Ayuntamiento de Ferrol, 1998: 191-202.

COLLIS, J. (1993): «Los celtas en Europa», en M. ALMAGRO-GORBEA y G. RUÍZ ZAPATERO (eds.). Los Celtas: Hispania y Europa. Madrid. Editorial Actas: 63-75.

COLLIS, J. (1996): «The origin and spread of the Celts», en Studia Celtica, XXX: 17-34.

COLLIS, J. (1996a): «Celts and politics», en P. GRAVES-BROWN, S. JONES \& C. GAMBLE (eds.), Cultural Identity and Archaeology. The construction of European Communities, T.A.G., Routledge, London: 167-178.

COLLIS, J. (1996b): «Urbanisation in Atlantic Europe in the Iron Age», Gallaecia, 14-15: 223-241.

COLLIS, J. (1997): «Celtic myths», en Antiquity, 71: 195-201.

CRIADO BOADO, F. (1993): «Límites y posibilidades de la Arqueología del Paisaje», SPAL, 2, Sevilla: 9-55.

CRIADO BOADO, F. et al. (1991): Arqueología del Paisaje, el área Bocelo-Furelos entre los tiempos megalíticos y medievales, Serie Arqueología/Investigación 6, Ed. Xunta de Galicia.

DE LA PEÑA SANTOS, A. (1992): «El primer milenio a.C. en el área gallega: génesis y desarrollo del mundo castreño a la luz de la Arqueología», en M. ALMAGRO-GORBEA y G. RUÍZ ZAPATERO (eds.), Paleoetnología de la Península Ibérica, Complutum 2-3, Madrid: 373-394.

«CUADERNOS DE ESTUDIOS GALLEGOS», Tomo XLVIII, Fascículo 114, Santiago 2001. 
DE LA PEÑA SANTOS, A. (1995): «La secuencia cultural del mundo castrexo galaico», en J.M. HIDALGO CUÑARRO (coord.), A Cultura Castrexa Galega a debate, Instituto de Estudos Tudenses, Vigo: 65-103.

DE LA PEÑA SANTOS, A. (1997): «Os pobos castrexos antes da conquista romana», en $O$ feito diferencial galego na Historia, Vol. I, Museo do Pobo Galego, Santiago de Compostela: 143-191.

DE LA PEÑA SANTOS, A.; VÁZQUEZ VARELA, J.M. (1996): «Aspectos de la génesis y evolución de la Cultura Castrexa de Galicia», Complutum, Extra 6(I), Madrid: 255-262.

DÍAZ SANTANA, B. (e.p.): «Arqueología y Política en Galicia: revisando la historiografía», en II Congreso Internacional sobre la Cultura Celta. Os celtas da Europa Atlántica, Noviembre de 1998, El Ferrol.

DÍAZ SANTANA, B. (2002): Los Celtas en Galicia. Arqueología y Politica en la creación de la Identidad Gallega. Noia, Toxosontos, Serie Keltia.

DÍAZ-ANDREU, M. (1995): «Archaeology and Nationalism in Spain», en P. L. KOHL \& C. FAWCETT (eds.), Nationalism, Politics and the practise of Archaeology, Cambridge University Press: 39-56.

DÍAZ-ANDREU, M. (1996): «Constructing identities through Culture. The Past in the forging Europe», en P. GRAVES-BROWN, S. JONES \& C. GAMBLE (eds.), Cultural Identity and Archaeology. The construction of European Communities, T.A.G., Routledge, London: 49-61.

DÍAZ-ANDREU, M. (1997): «Nación e internacionalización. La Arqueología en España en las tres primeras décadas del siglo XX», en $\mathrm{G}$. MORA \& M. DÍAZ-ANDREU (eds.), La cristalización del pasado: génesis y desarrollo del marco institucional de la Arqueología en España, Málaga: 403-416.

«CUADERNOS DE ESTUDIOS GALLEGOS», Tomo XLVIII, Fascículo 114, Santiago 2001. 
DÍAZ-ANDREU, M. (1997a): «Prehistoria y Franquismo», en G. MORA \& M. DÍAZ-ANDREU (eds.), La cristalización del pasado: génesis y desarrollo del marco institucional de la Arqueología en España, Málaga: 547-552.

DÍAZ-ANDREU, M.; MORA, G. (1995): «Arqueología y Política: el desarrollo de la Arqueología española en su contexto histórico», en $T P$, 52 (I): 25-38.

DÍAZ-ANDREU, M.; CHAMPION, T. (1996): «Nationalism and Archaeology in Europe: an introduction», en M. DÍAZ-ANDREU \& T. CHAMPION (eds.), Nationalism and Archaeology in Europe, University College of London Press: 1-23.

DÍAZ-ANDREU, M.; MORA, G. (1997): «La historiografía española sobre Arqueología: panorama actual de la investigación», en G. MORA \& M. DÍAZ-ANDREU (eds.), La cristalización del pasado: génesis y desarrollo del marco institucional de la Arqueología en España, Málaga: 9-18.

FERNÁNDEZ-POSSE, M. D. (1998): La investigación Protohistórica en la Meseta y Galicia, Arqueología Prehistórica 1, Madrid, Ed. Síntesis.

GARCÍA CÁRCEL, R. (1994): «La manipulación de la memoria histórica en el Nacionalismo Español», en Manuscrits, $n^{\circ} 12$ : 175-181.

GARCÍA FERNÁNDEZ-ALBALAT, B. (1990): Guerra y religión en la Gallaecia y Lusitania antiguas, Ed. Do Castro, Sada, 247 pp.

GARCÍA FERNÁNDEZ-ALBALAT, B. (1997): «Sociedade e categorías sociais na Gallaecia antiga», en Sociedade e categorías sociais na Historia de Galiza, Editorial A Nosa Terra, Col. A Nosa Historia 6, Vigo: 10-16.

GARCÍA QUINTELA, M. V. (1996): «Las puertas del Infierno y el Río del Olvido (un tema mítico céltico en la etnografía ibérica de Estrabón)», Gallaecia, 15: 145-157. 
GARCÍA Y BELLIDO, A. (1946/47): «Sobre un tipo de casa-choza gallega», C.E.G., nº2: 490-492.

GONZÁLEZ REBOREDO, X. M. (1990): «Elementos de identidade nos historiadores e etnógrafos galegos da primeira metade do século $\mathrm{XX} »$, en Identidade e territorio, Centenario de Otero Pedrayo, Consello da Cultura Galega: 215-220.

GONZÁLEZ-PARDO, I. M. (1983): «El anticeltismo de Francisco Martins Sarmento», en I Coloquio Galaico-Minhoto, Vol. II, Ponte de Lima 1982. Edit. Braga: 45-109.

HERNANDO GONZALO, A. (1992): «Los enfoques teóricos en Arqueología», SPAL, 1: 11-35.

JAMES, S. (1998): "Celts, politics and motivation in Archaeology», Antiquity, 72 (98): 209-213.

JAMES, S. (1999): The Atlantic Celts. Ancient people or modern invention?, British Museum Press, London, 160 pp.

JENSEN, O. W. (1997): «When Archaeology meets Clio. A critical reflection on writing the History of Archaeology», en Archaeological Review from Cambridge, vol.14 (2): 79-91.

JIMÉNEZ DÍEZ, J. A. (1993): Historiografía de la Pre y Protohistoria de la Península Ibérica en el siglo XIX, tesis doctoral inédita, Universidad Complutense.

JIMÉNEZ DÍEZ, J. A. (1996): «Del mito a la Prehistoria en la Historia de España: aproximación historiográfica (1841-1900)», en Complutum, 7: 265-273.

JORGE DÍAS, S. (1946/47): «Sobre las construcciones circulares del Noroeste de la Península Ibérica y las Citanias», C.E.G., nº 2: 173-194.

«CUADERNOS DE ESTUDIOS GALLEGOS», Tomo XLVIII, Fascículo 114, Santiago 2001. 
JUEGA PUIG, J. (1995): «La implantación de un mito: el celtismo en Galicia», en J. M. HIDALGO CUÑARRO (ed.) A Cultura Castrexa galega a debate, Vigo: 43-61.

KOHL, P. L. \& FAWCETT, C. (1995): «Archaeology in the service of the State: theoretical considerations», en P. L. KOHL \& C. FAWCETT (eds.), Nationalism, Politics and the practise of Archaeology, Cambridge University Press: 3-20.

LÓPEZ CUEVILLAS, F. (1946/47): «Armería posthallstáttica del Noroeste Hispánico», C.E.G., nº 2: 543-589.

LÓPEZ CUEVILLAS, F. (1925): A Edade do Ferro na Galiza, Revista Nós, A Cruña.

LÓPEZ CUEVILLAS, F. (1949): «Sobre las relaciones con Bretaña», C.E.G., Miscelánea, $\mathrm{n}^{\circ}$ 4: 439-440.

LÓPEZ CUEVILLAS, F. (1950a): «Las fíbulas castreñas y su significado etnológico», C.E.G., nº 5: 5-19.

LÓPEZ CUEVILLAS, F. (1956): «Antropología física y Prehistoria gallegas», en C.E.G., T. XI: 455-456.

LÓPEZ CUEVILLAS, F. (1956): «Antropología física y Prehistoria Gallegas», C.E.G., n 11: 455-456.

LÓPEZ CUEVILLAS, F.; BOUZA BREY, F. (1827): «Bibliografía da Prehistoria Galega», Publicazóns do Seminario de Estudos Galegos, Seizón de Prehistoria, Revista Nós, A Cruña: 18 págs.

LÓPEZ CUEVILLAS, F.; BOUZA BREY, F. (1929): Os Oestrimnios, os Saefes e a Ofiolatría en Galiza, Arquivos do Seminario de Estudos Galegos-II-1929, Seizón de Prehistoria, Nós, A Cruña.

LÓPEZ CUEVILLAS, F.; LORENZO FERNÁNDEZ, J. (1946/47): «Las habitaciones de los castros», C.E.G., n ${ }^{\circ}$ : 7-73.

«CUADERNOS DE ESTUDIOS GALLEGOS», Tomo XLVIII, Fascículo 114, Santiago 2001. 
LÓPEZ CUEVILLAS, F.; RUI DE SERPA PINTO, (1933/34): «Estudos sobre a Edade do Ferro no Noroeste Peninsuar. As tribus e sua constitución política», en Arquivos do Seminario de Estudos Galegos, 6, Santiago de Compostela: 261-294.

LÓPEZ GARCÍA, J.C. (1997): «A influencia do celtismo na obra de José Villaamil y Castro», en Gallaecia, 16, Santiago de Compostela: 97-110.

LÓPEZ PAZ, M. P.; PEREIRA MENAUT, G. (1995/96): «La tierra y los hombres: paisaje político, paisaje histórico», Stud. Hist., Historia Antigua, 13-14, Salamanca: 39-60.

LORRIO ALVARADO, A. (1991): " Los celtas en el Noroeste», Los celtas en la Península Ibérica, Revista de Arqueología, Zugarto Ediciones, Madrid: 26-35.

LORRIO, A. J. (1993): «El armamento de los Celtas hispanos», en M. ALMAGRO-GORBEA \& G. RUÍZ ZAPATERO (eds.), Los Celtas: Hispania y Europa, Ed. Actas, Madrid: 285-326.

LUENGO MARTÍNEZ, J. M. (1971): «Lauda funeraria del siglo XV con supervivencias célticas en el Museo Histórico-Arqueológico de La Coruña», C.E.G., n 26, fasc. 78: 87-94.

MACÍAS Y GARCÍA, M. (1892): De Galicia. Discursos de carácter regional pronunciados en las ciudades de la Coruña, Orense y Vigo, Andrés Martínez editor, La Coruña, 1892.

MÁIZ SUAREZ, R. (1997): A idea de Nación, Ed. Xerais de Galicia, Vigo, 1997.

MÁIZ SUAREZ, R. (1984): O rexionalismo galego: organización e ideoloxía, (1886-1907), Publicación do Seminario de Estudos Galegos, A Coruña, 1984.

MANSILLA CASTAÑO, A. M. (1997): «La negación del pasado de los otros: Arqueología y xenofobia», en T.P., 54 (I): 21-34. 
MARCO SIMÓN, F. (1993): «La religiosidad en la Céltica Hispana», en M. ALMAGRO-GORBEA \& G. RUÍZ ZAPATERO (eds.), Los Celtas: Hispania y Europa, Ed. Actas, Madrid: 477-512.

MARCO SIMÓN, F. (1998): «La religión en la Céltica Hispana», en UNIÓN CULTURAL ARQUEOLÓGICA (eds.), Celtas y celtíberos. Realidad o leyenda, Madrid: 159-169.

MARTÍNEZ MURGUÍA, M. (1882): Estudios sobre la propiedad territorial en Galicia, Santiago de Compostela.

MARTÍNEZ MURGUÍA, M. (1886): Los Precursores, Latorre y Martínez (eds.), Imprenta La Voz de Galicia ( $2^{\circ}$ facsímil y $5^{\text {a }}$ ed.), La Coruña.

MARTÍNEZ MURGUÍA, M. (1888): Galicia, en Biblioteca de Autores Galegos, Sálvora, 1985.

MARTÍNEZ MURGUÍA, M.; VICETTO, B. (1886): Historia de Galicia, en Real Academia Gallega, reproducción facsímil en Gran enciclopedia Vasca, Vol. I, 1980.

PARCERO OUBIÑA, C. (1993): «Aproximación al espacio social en el Mundo Castreño», Actas del XXII Congreso Nacional de Arqueología, Vigo: 185-188.

PARCERO OUBIÑA, C. (1995): «Elementos para el estudio de los paisajes castreños del Noroeste Peninsular», T.P., 52 (I), Madrid: 127-144.

PARCERO OUBIÑA, C. (1997a): Documentación de un entorno castreño. Trabajos arqueológicos en el área de Cameixa, TAPA 1, Grupo de Investigación en Arqueología del Paisaje, Santiago de Compostela.

PARCERO OUBIÑA, C. (1997b): «The invisible warrior: warfare and Archaeology in the Indoeuropean Bronze Age», en F. CRIADO \& C. PARCERO (eds.), Landscape. Archaeology. Heritage, TAPA 2, GiArPa, Santiago de Compostela: 35-40.

«CUADERNOS DE ESTUDIOS GALLEGOS», Tomo XLVIII, Fascículo 114, Santiago 2001. 
PARCERO OUBIÑA, C.; CRIADO BOADO, F.; SANTOS ESTÉVEZ, M. (1998): «Rewriting landscape: incorporating sacred landscapes into cultural traditions», World Archaeology, Vol. 30 (I): 159-176.

PEIRÓ MARTÍN I.; PASAMAR ALZURIA, G. (1989/90): «El nacimiento en España de la Arqueología y la Prehistoria (Academicismo y Profesionalización) (1856-1936), Kalathos 9-10, Revista del S.A.E.T., Teruel: 9-30.

PENA GRAÑA, A. (1994): «O territorio e as categorías sociais na Gallaecia Antiga: un matrimonio entre a Terra (Treba) e a Deusa Nai (Mater)», Anuario Brigantino, 17: 33-78.

PENA GRAÑA, A. J. (1993): »Un rexistro arqueolóxico das institucións nos territorios políticos do Noroeste Peninsullar na koiné cultural (céltica) do Ferro Atlántico: Treba, Trebopala, Oenach/Forum», Actas del XXII Congreso Nacional de Arqueología, Vigo: 159-164.

PEREIRA GONZÁLEZ, F. (1996): «Unha contribución ó estudio da Historia da Arqueoloxía galega: o emprego da información arqueolóxica en Galicia (1800-1922)», en Gallaecia, 14-15, Santiago de Compostela: 7-29.

PEREIRA GONZÁLEZ, F. (1996): «O pensamento arqueolóxico en antropolóxico de Leandro Saralegui y Medina (1839-1910)», C.E.G., 1996: 9-36.

PEREIRA GONZÁLEZ, F. (1997): «As opinión sobre a Humanidade Primitiva na Galiza do Século XX e as súas relacións coa información arqueolóxica», en Gallaecia, 16, Santiago de Compostela: 71-95.

PEREIRA GONZÁLEZ, F. (2000): Gallaecia, 19: 311-333.

PEREIRA MENAUT, G. (1992): «Aproximación crítica al estudio de la etnogénesis: la experiencia de Gallaecia», M. ALMAGRO-GORBEA y G. RUÍZ ZAPATERO (eds.), Paleoetnología de la Península Ibérica, Complutum 2-3, Madrid: 35-43. 
PEREIRA MENAUT, G. (1994): «Sobre la función del pasado histórico en los movimientos nacionalistas», en P. SÁEZ y S. ORDÓÑEZ (eds.), Homenaje al Profesor Presedo, Secretariado de Publicaciones Universitarias de Sevilla: 851-862.

PEREIRA MENAUT, G. (1997): «Un pobo e unha natio moi particulares», en O feito Diferencial Galego na Historia, Vol. I, Museo do Pobo Galego, Santiago de Compostela:237-249.

PRADO FERNÁNDEZ, O. (1997): «El Seminario de Estudos Galegos: génesis, aportación y desarrollo», en G. MORA y M. DÍAZ-ANDREU, La cristalización de pasado: génesis y desarrollo del marco institucional de la Arqueología en España, 1997, Málaga: 457-461.

RENALES CORTÉS, J. (1996): Celtismo y Literatura Gallega. La obra de Benito Vicetto y su entorno Literario, Ed. Xunta de Galicia, Santiago de Compostela, 1996.

RENFREW, C. (1996): «Prehistory and the identity of Europe. Or, Don't let's be beastly to the Hungarians», P. GRAVES-BROWN, S. JONES \& C. GMABLE (eds.), Cultural Identity and Archaeology: the construction of European Communities, T.A.G., Routledge, London: 125-137.

ROJO SALGADO, A. (1989): O Galeguismo a través dos seus textos e documentos políticos, Consellería de Cultura e Deportes, Revista Coordenadas - Monografías, 9, Santiago de Compostela, Abril, 1989.

ROMERO MASIÁ, A. (1984/85): «Os castros: recoñecemento e catalogación», C.E.G., nº 35, fasc. 100: 31-62.

RUÍZ ZAPATERO, G. (2001): «¿Quiénes fueron los celtas? Disipando la niebla: Mitología de un collage histórico», en VV. AA., Celtas y Vetones, Ávila: Edita Excma. Diputación Provincial de Avila.

SARALEGUI Y MEDINA, L. (1894): La época céltica en Galicia, Tercera edición, Librería de R. Pita, Ferrol.

«CUADERNOS DE ESTUDIOS GALLEGOS», Tomo XLVIII, Fascículo 114, Santiago 2001. 
TORRES, C. (1949): «Límites geográficos de Galicia en los siglos IV y $\mathrm{V} »$, C.E.G., $\mathrm{N}^{\circ} 4: 366$ y ss.

TRIGGER, B. G. (1992): Historia del Pensamiento Arqueológico, Ed. Crítica, 1992, 475 pp.

TRIGGER, B. G. (1995): «Romanticism, Nationalism and the practise of Archaeology», P. L. FOHL \& C. FAWCETT (eds.), Nationalism, Politics and the Practise of Archaeology, Cambridge University Press: 263-279.

VEREA Y AGUIAR, J. (1838): Historia de Galicia. Primera parte, que comprende los orígines y estado de los pueblos septentrionales y occidentales de la España antes de su conquista por los romanos, Imprenta Nicasio Taxonera, Ferrol.

VICETTO, B. (1865): Historia de Galicia, Imprenta Nicasio Taxonera, Ferrol.

VILLAAMIL Y CASTRO, J. (1873). Antigüedades prehistóricas y célticas de Galicia. Parte primera, Lugo.

VILLAAMIL Y CASTRO, J. (1875): Ensayo de un catálogo sistemático y crítico de los libros que tratan de Galicia, Madrid. 